\title{
Reactive Power Optimization in Integrated Electricity and Gas Systems
}

\author{
Pengfei Zhao, Student Member, IEEE, Chenghong Gu, Member, IEEE, Yue Xiang, Member, IEEE, \\ Xin Zhang, Senior Member, IEEE, Yichen Shen and Shuangqi Li, Student Member, IEEE
}

\begin{abstract}
Volt/VAR optimization (VVO) is one important operation in distribution systems to maintain acceptable voltage profiles. However, the high penetration of renewable generation poses severe challenges to VVO, leading to voltage deviation and fluctuation. This is further complicated by the growing coupling between electricity and natural gas systems.

To resolve unacceptable voltage deviation under energy system interdependency, this paper proposes a co-optimization of VVO for an integrated electricity and gas system (IEGS) with uncertain renewable generation. A two-stage data-driven distributionally robust optimization (DRO) is developed to model the coordinated optimization problem, which determines two-stage VVO and operation schemes with dispatch and corrective adjustment through active power regulation and reactive power support in both dayahead and real-time stage. A semidefinite programming is reformulated to ensure the tractability and the proposed problem is solved by a constraint generation framework. Simulation studies are conducted on a 33-bus-6-node IEGS. Case studies demonstrate that the interdependency between electricity and gas systems reduces siginificant operation cost and voltage rise. It thus can benefit integrated system operators with a powerful operation tool to manage systems with fewer costs but integrate more renewable energy while maintaining high supply quality.
\end{abstract}

Index Terms -Data-driven optimization, distributionally robust optimization, integrated electricity and gas system, two-stage framework, volt/VAR optimization.

\section{NOMENCLATURE}

\section{A. Indices and sets}
$t, T$ Index and set for time periods.
$b, B \quad$ Index and set for electricity buses.
$i_{e}, I_{e} \quad$ Index and set for traditional distributed generators (DG).
$i_{g}, I_{g} \quad$ Index and set for natural gas sources.
$g t, G T \quad$ Index and set for gas turbines.
$j, J \quad$ Index and set for renewable power generators.
$l_{e}, L_{e} \quad$ Index and set for power lines.
$l_{g}, L_{g} \quad$ Index and set for gas pipelines.
$k_{e}, K_{e} \quad$ Index and set for electric loads.

Manuscript received January 17, 2020; revised March 12 and April 29, 2020; accepted May 2, 2020.

P. Zhao, C.Gu (corresponding author), Y. Shen and S. Li are with the Department of Electronic \& Electrical Engineering, University of Bath, Bath,

UK. (email: P.Zhao@bath.ac.uk; C.Gu@bath.ac.uk; Y.Shen@bath.ac.uk; S.Li@bath.ac.uk).

Y. Xiang is with College of Electrical Engineering, Sichuan University, China. (email: xiang@ @scu.edu.cn)

X. Zhang is with Centre for Energy Systems and Strategy, Cranfield University, Cranfield, UK. (email: xin.zhang@ cranfield.ac.uk). $k_{g}, K_{g} \quad$ Index and set for gas loads.

\section{B. Parameters}

$w_{1}^{V}, w_{1}^{E} \quad$ Weighting coefficients for voltage regulation and economic dispatch oriented subobjectives in the first stage.

$w_{2}^{V}, w_{2}^{E} \quad$ Weighting coefficients for voltage regulation and economic dispatch oriented subobjectives in the second stage.

$\pi_{v} \quad$ the penalty cost coefficient for penalizing the voltage deviation.

$V_{b}^{r e f} \quad$ Nominal voltage magnitude.

$\lambda_{\text {sub }}^{a}, \lambda_{\text {sub }}^{r} \quad$ Unit cost for active and reactive power supplied from upper market.

$\lambda_{i_{e}}^{a}, \lambda_{i_{e}}^{b}, \lambda_{i_{e}}^{c} \quad$ Cost coefficients for generation of traditional $\mathrm{DG} i_{e}$.

$\lambda_{i_{g}} \quad$ Cost coefficient for generation of natural gas source $i_{g}$.

$\lambda_{i_{e}}^{+}, \lambda_{i_{e}}^{-} \quad$ Cost coefficient for up and down reserve of traditional DG $i_{e}$.

$\lambda_{i_{e}}^{r e}, \lambda_{j}^{r e} \quad$ Regulation cost coefficient for traditional DG $i_{e}$ and renewable power generator $\mathrm{j}$.

$\lambda_{k_{e}}^{l s}, \lambda_{k_{g}}^{l s} \quad$ Penalty cost coefficient for electricity and gas load shedding.

$P_{\text {sub,max }} \quad$ Maximum active power transfer of substation.

$R_{i_{e}}^{+}, R_{i_{e}}^{-} \quad$ Maximum up and down reserve capacity of traditional DG $i_{e}$ at time $t$.

$R_{g t}^{+}, R_{g t}^{-} \quad$ Maximum up and down reserve capacity of gas turbine $g t$ at time $\mathrm{t}$.

$P_{i_{e}, \max }, \quad$ Maximum and minimum output of

$P_{i_{e}, \text { min }} \quad$ tradiational DG $i_{e}$.

$P_{i_{g}, \max }, \quad$ Maximum and minimum output of natural $P_{i_{g}, \min } \quad$ gas source $i_{g}$.

$P_{g t, \max }, \quad$ Maximum and minimum output of gas

$P_{g t, \min } \quad$ turbine $g t$.

$V_{b, \text { max }}, V_{b, \text { mir }}$ Maximum and minimum voltage limit.

$\delta^{\text {OLTC }} \quad$ Size of change for each step in OLTC tap position.

$n T P_{\max }^{O L T C} \quad$ Maximum allowed number of switching operations of OLTC. 


\begin{tabular}{|c|c|}
\hline$\omega_{j, t}^{P, s}$ & $\begin{array}{l}\text { Forecasted active power output of renewable } \\
\text { power generator } j \text { at time } t \text {. }\end{array}$ \\
\hline$u_{P V}$ & $\begin{array}{l}\text { Associated coefficient for connecting active } \\
\text { and reactive } \mathrm{PV} \text { power. }\end{array}$ \\
\hline$P F_{p v, \min }$ & Minimum power factor of PV system $p v$. \\
\hline$Q_{c b}^{c a p}$ & $\begin{array}{l}\text { Reactive power capability for capacitor bank } \\
c b \text {. }\end{array}$ \\
\hline$V_{0}$ & Reference voltage magnitude. \\
\hline $\begin{array}{l}f_{l_{e}, \max }^{a,} \\
f_{l_{e}, \max }^{r,}\end{array}$ & $\begin{array}{l}\text { Maximum active and reactive power flow of } \\
\text { line } l_{e} \text {. }\end{array}$ \\
\hline $\begin{array}{l}P_{k_{e}, t} \\
Q_{k_{e}, t}, P_{k_{g}, t}\end{array}$ & $\begin{array}{l}\text { Active and reactive electricity load and gas } \\
\text { load at time } t \text {. }\end{array}$ \\
\hline $\begin{array}{l}P r_{l_{g}, \max } \\
P r_{l_{g}, \min }\end{array}$ & $\begin{array}{l}\text { Maximum and minimum gas pressure of gas } \\
\text { pipeline } l_{g} \text {. }\end{array}$ \\
\hline$\gamma_{l_{g}}$ & Coefficient for Weymouth equation. \\
\hline$f_{l_{g}, \max }$ & Maximum gas flow of pipeline $l_{g}$. \\
\hline $\begin{array}{l}P_{k_{e}, \max }^{l s} \\
P_{k_{g}, \max }^{l s}\end{array}$ & $\begin{array}{l}\text { Maximum electricity and gas load shedding } \\
\text { at time } t \text {. }\end{array}$ \\
\hline$\eta_{i_{e}, t}, \eta_{g t, t}$ & $\begin{array}{l}\text { Participation factor for reserves of traditional } \\
\text { DG } i_{e} \text { and gas turbine } g t \text { at time } t \text {. }\end{array}$ \\
\hline
\end{tabular}

C. Variables and functions

$P_{\text {sub }, t}^{s}, \quad$ Scheduled active and reactive power supply

$Q_{\text {sub }, t}^{S} \quad$ from upper market.

$P_{\text {sub }, t}^{r e}, \quad$ Regulated active and reactive power supply

$Q_{\text {sub }, t}^{r e} \quad$ from upper market.

$P_{i_{e}, t}^{S}, P_{i_{e}, t}^{r e} \quad$ Scheduled and regulated output of traditional DG $i_{e}$ at time $t$.

$P_{i_{g}, t}^{s}, P_{i_{g}, t}^{r e} \quad$ Scheduled and regulated output of natural gas source $i_{g}$ at time $t$.

$r_{i_{e}, t}^{+}, r_{i_{e}, t}^{-} \quad \mathrm{Up}$ and down reserve of traditional DG $i_{e}$ at time $t$.

$r_{g t, t}^{+}, r_{g t, t}^{-} \quad$ Up and down reserve of gas turbine $g t$ at time t.

$V_{b, t}^{s}, V_{b, t}^{r e} \quad$ Scheduled and regulated voltage of bus $\mathrm{b}$ at time $t$.

$V_{s u b, t}^{s}, V_{s u b, t}^{r e} \quad$ Scheduled and regulated voltage of substation at time $t$.

$T P_{t}^{s, O L T C}, \quad$ Scheduled and regulated tap position of

$T P_{t}^{r e, O L T C} \quad$ OLTC at time $t$.

$\omega_{j, t}^{Q, S}, \omega_{j, t}^{Q, r e} \quad$ Scheduled and regulated reactive power output of renewable power generator $j$ at time $t$.

$u_{c b, t}^{s}, u_{c b, t}^{r e} \quad$ Scheduled and regulated switch status for capacitor bank $c b$ at time $t$.

$Q_{c b, t}^{S}, Q_{c b, t}^{r e} \quad$ Scheduled and regulated reactive power output for capacitor bank $c b$ at time $t$.

$V_{b, t}^{s, i n i}, \quad$ Scheduled voltage magnitude for initial and $V_{b, t}^{s, t e r} \quad$ terminal nodes.

\begin{tabular}{|c|c|}
\hline $\begin{array}{l}V_{b, t}^{r e, i n i}, \\
V_{b, t e r}^{r e t}\end{array}$ & $\begin{array}{l}\text { Regulated voltage magnitude for initial and } \\
\text { terminal nodes. }\end{array}$ \\
\hline$f_{l_{e}, t}^{a, t}, f_{l_{e}, t}^{r, s}$ & $\begin{array}{l}\text { Scheduled active and reactive power flow at } \\
\text { time } t \text {. }\end{array}$ \\
\hline$f_{l_{e}, t}^{a, r e}, f_{l_{e}, t}^{r, r e}$ & $\begin{array}{l}\text { Regulated active and reactive power flow at } \\
\text { time } t \text {. }\end{array}$ \\
\hline$f_{l_{g}, t}^{i n i}, f_{l_{g}, t}^{t e r}$ & $\begin{array}{l}\text { Gas flow from initial node and to terminal } \\
\text { node of pipeline } l_{g} \text { at time } t \text {. }\end{array}$ \\
\hline $\operatorname{Pr}_{l_{g}, t}^{S}$, & Scheduled and regulated gas pressure of gas \\
\hline$P r_{l_{g}, t}^{r e}$ & pipeline $l_{g}$ at time $t$. \\
\hline $\begin{array}{l}P r_{l_{g}, t}^{s, i n i} \\
P r_{l_{g}, t}^{s, t e r}\end{array}$ & $\begin{array}{l}\text { Scheduled gas pressure of initial and terminal } \\
\text { nodes of pipeline } l_{g} \text { at time } t \text {. }\end{array}$ \\
\hline $\begin{array}{l}P r_{l_{g}, t}^{r e, i n i}, \\
P r_{l_{g}, t}^{r e, t e r}\end{array}$ & $\begin{array}{l}\text { Regulated gas pressure of initial and terminal } \\
\text { nodes of pipeline } l_{g} \text { at time } t \text {. }\end{array}$ \\
\hline$P_{k_{e}, t}^{l s}, P_{k_{g}, t}^{l s}$ & Electricity and gas load shedding at time $t$. \\
\hline $\begin{array}{l}x, y \\
D . \quad U\end{array}$ & $\begin{array}{l}\text { Vectors of first and second stage variables. } \\
\text { rtainty }\end{array}$ \\
\hline$\xi_{j, t}$ & $\begin{array}{l}\text { Uncertainty of renewable power forecast of } j \\
\text { at time } t \text {. }\end{array}$ \\
\hline$D_{\xi_{j, t}}$ & $\begin{array}{l}\text { Ambiguity set for renewable power } \\
\text { uncertainty. }\end{array}$ \\
\hline$\mu_{\xi_{j, t}} \Sigma_{\xi_{j, t}}$ & $\begin{array}{l}\text { Mean vector and covariance matrix for } \\
\text { renewable forecast uncertainty. }\end{array}$ \\
\hline$\Theta$ & Second moment matrix. \\
\hline & Polyhedral set of extreme points. \\
\hline
\end{tabular}

\section{INTRODUCTION}

Volt-Var optimization (VVO) is one primary function in the distribution management system to maintain voltage in an acceptable range by optimally coordinating equipment, e.g., capacitor banks, on-load tap changers (OLTC) and voltage regulators [1-6]. Paper [2] proposes a deterministic VVO as mixed-integer quadratic programming to control voltage and VAR devices for day-ahead operation. Considerable loss reduction and total demand reduction are achieved through the proposed VVO. To achieve energy savings and peak demand reduction through voltage reduction, a multi-objective VVO is proposed in [3] using a nondominated sorting genetic algorithm. Paper [4] proposes a three-phase distribution system considering unbalancing with battery storage providing reactive power. Both power loss and energy purchase cost are minimized. In [5], to extend the life of distribution system transformers, a detailed model of life loss is proposed to estimate the ageing reduction of transformers under VVO.

The penetration of renewable energy has dramatically increased over the past decade. However, due to its variable and intermittent nature, it poses operational and security challenges to VVO by affecting normal operations of OLTCs and capacitor banks [6-8]. Existing literature has considered renewable power uncertainties in designing economic and reliable periodical equipment scheduling plans. A two-stage 
chance-constrained VVO is employed to handle the uncertainties of distributed generation and load demand [6]. Paper [7] proposes a chance-constrained optimization to model the randomness of renewable energy and minimize feeder power losses while avoiding voltage violations, solved by a gradient descent based algorithm. A hierarchical robust optimization (RO) is adopted for coordinating reactive compensators to guarantee voltage magnitudes [8]. This reactive power optimization is formulated to mixed-integer convex-based programming based on the conic relaxation of the branch flow. And a modified column-and-constraint generation algorithm based on the second-order cone programming is employed to solve the problem.

The interdependency of multi-energy systems, electricity, natural gas, and heating/cooling, is also becoming markedly common, which has many implications for VVO. Research on integrated electricity and gas systems (IEGS) is widely investigated in traditional problems, e.g., modelling, operation and planning [9-11]. However, high renewable integration brings security and operational challenges to managing IEGS, particularly to system voltage, due to intermittency and fluctuation, [11-13]. To hedge against renewable power uncertainty in IEGS, deterministic optimization, two-stage and multi-stage stochastic optimization (SO) have been widely used [12]. For example, paper [13] proposes a robust securityconstrained unit commitment in IEGS considering distributed natural gas storage to enhance operational reliability.

Overall, RO and SO are the two main approaches to handle uncertainties from growing renewable energy resources for both VVO and IEGS operation problems. As for SO, it either assumes an explicit distribution for random variable or requires a large number of data samples. It is prone to causing errors when the historical data is not adequately sufficient to represent true distributions and inevitably leads to high computational burden if a large number of data samples are used. As for RO, it does not require an exact probability distribution but constrains uncertain variables in a predefined uncertainty set. Therefore, it is of necessity to handle the uncertainty through a relative less conservative optimization technique without requiring large datasets. As a promising optimization method to handle uncertainties, distributionally robust optimization (DRO) inherits the advantages of both RO and SO, overcoming the explicit assumptions on probability distributions of $\mathrm{SO}$ and over-conservatism of RO [14-17].

A security-constrained two-stage ED with renewable power uncertainty modelled by DRO is designed in [18]. The segregated linear decision rule is used to affinely approximate the decision variables after the first time period which reduces the computational burden. Paper [19] investigates a DRO for IEGS considering the uncertainties of electricity and gas loads. Price-Based demand response is considered to improve energy efficiency and economic benefits. DRO can provide a less conservative solution for VVO problem when capturing renewable uncertainties and thus mitigate the impact on voltage deviations caused by renewable uncertainties. In addition, the two-stage framework contains both day-ahead and real-time framework, which provides flexible measures for system operators with adjustment capability.
This paper proposes a novel coordinated two-stage multiobjective optimization for voltage control in economic dispatch (ED), considering uncertain renewable generation and multivector energy system integration. The two-stage voltage constrained optimization is referred to as TS-VCO for simplicity. The optimal voltage is achieved through efficiently coordinating the operation of OLTCs, photovoltaic (PV) systems, and shunt capacitor banks. In the first stage, based on historical PV output, an initial day-ahead operation plan for traditional DGs, natural gas sources, OLTCs and capacitor banks is produced to maintain voltage and minimize daily operation cost. In the second stage, after the realization of uncertain PV output, the recourse action is developed to control voltage controlling those devices, meanwhile minimizing system operation cost in real time. The original TS-VCO is transformed into a conic tractable form with the dual formulation and solved by constraint generation algorithm (CGA). Case studies demonstrate that voltage control devices and dispatchable generators can be optimally controlled and coordinated to realise the designed objectives.

The main contributions of this paper are as follows:

1) To the best of authors' knowledge, none existing work has investigated VVO in an IEGS, where this paper is the first such effort to fill the research gap. The strong coupling of power and gas infrastructure and tight interdependency between two systems are considered.

2) It develops a multi-objective coordinated optimization for maintaining acceptable voltage while considering system operation cost of IEGS, which ensures system security and economic performance.

3) The two-stage DRO approach is first applied in VVO, which provides less-conservative results using RO and requires fewer data samples. It can handle renewable uncertainties effectively, providing flexible measures for IEGS operators.

The rest of this paper is organized as follows. Section II presents objective function and system constraints of TS-VCO. The DRO method and associated reformulations are presented in section III. Section IV demonstrates case studies and the performance of the TS-VCO. Section V concludes this paper.

\section{SYSTEM MODELLING}

The proposed TS-VCO contains i) day-ahead cooptimization that restricts voltages for all buses while scheduling traditional DGs and natural gas sources, and ii) realtime recourse action that regulates voltage and redispatches generators considering PV output uncertainty.

The first-stage problem in (1) is to simultaneously minimize i) the voltage deviation for all buses, $\Gamma_{1}^{V}$ in (2) and ii) the cost of generation and reserve capacity, $\Gamma_{1}^{E}$ in (3), respectively. $\Gamma_{1}^{V}$ represents the total voltage deviation for all buses in the entire time horizon. $\Gamma_{1}^{E}$ includes i) generation cost of traditional DGs and natural gas sources and ii) reserve cost of traditional DGs. The generation cost function of traditional DGs is quadratic with coefficients $a_{i}, b_{i}$ and $c_{i}$. The weighting coefficients $w_{1}^{V}$ and $w_{1}^{E}$ represent the priorities that TS-VCO have on $\Gamma_{1}^{V}$ and $\Gamma_{1}^{E}$, respectively. It should be noted that the penalty cost coefficient $\pi_{v}$ is applied for penalizing the voltage deviation on 
each bus [20]. The penalty cost coefficient enables to transform voltage deviation to momentary lost which can be combined with operation cost.

$$
\begin{gathered}
\Gamma_{1}=\min w_{1}^{V} \pi_{v} \Gamma_{1}^{V}+w_{1}^{E} \Gamma_{1}^{E} \\
\Gamma_{1}^{V}=\min \sum_{b \in B, t \in T}\left|V_{b, t}^{s}-V_{b}^{r e f}\right| \\
\Gamma_{1}^{E}=\min \sum_{i_{e} \in I_{e}, i_{g} \in I_{g}, t \in T} \lambda_{s u b}^{a} P_{s u b, t}^{s}+\lambda_{s u b}^{r} Q_{s u b, t}^{s} \\
+\lambda_{i_{e}}^{a} P_{i_{e}, t}^{s}+\lambda_{e_{e}}^{b} P_{i_{e}, t}^{s}+\lambda_{i_{e}}^{c} \\
+\lambda_{i_{g}} P_{i_{g}, t}^{s}+\lambda_{i_{e}}^{+} r_{i_{e}, t}^{+}+\lambda_{i_{e}}^{-} r_{i_{e}, t}^{-}
\end{gathered}
$$

Similar to (1)-(3), (4)-(6) are the second-stage overall objective and sub-objectives. The second-stage optimization considers load shedding to keep the system balance under fluctuation caused by renewable uncertainties. It should be noted that $\Gamma_{2}^{E}$ contains i) the penalty cost for PV curtailment, ii) regulated generation cost of traditional DGs and natural gas sources and iii) electricity and gas load shedding cost.

$$
\begin{gathered}
\Gamma_{2}=\min w_{2}^{V} \pi_{v} \Gamma_{2}^{V}+\left(1-w_{2}^{V}\right) \Gamma_{2}^{E} \\
\Gamma_{2}^{V}=\min \sum_{b \in B, t \in T}^{E}\left|V_{b, t}^{r e}-V_{b}^{r e f}\right| \\
\Gamma_{i_{e} \in I_{e}, i_{g} \in I_{g}, t \in T, k_{e} \in K_{e}, k_{g} \in K_{g}} \lambda_{s u b}^{a} P_{s u b, t}^{r e} \\
+\lambda_{s u b}^{r} Q_{s u b, t}^{r e}+\lambda_{j}^{r e}\left|\omega_{j, t}^{s}-\xi_{j, t}\right| \\
+\lambda_{i_{e}}^{r e}\left|P_{i_{e}, t}^{s}-P_{i_{e}, t}^{r e}\right|+\lambda_{i_{e}}^{r e} \mid P_{i_{g}, t}^{s} \\
-P_{i_{g}, t}^{r e} \mid+\lambda_{k_{e}}^{l s} P_{k_{e}, t}^{l s}+\lambda_{k_{g}}^{l s} P_{k_{g}, t}^{l s}
\end{gathered}
$$

\section{A. Day-ahead VVO}

In the first stage, the day-ahead optimization is based on the forecasted renewable output before its uncertainty realised. Equations (7)-(29) represent the first-stage constraints. The active and reactive power for substation injected from the upper level is limited in (7) and (8). The up and down reserve capacity for traditional DGs and gas turbines is constrained in (9) and (10). In the distribution network, the proposed day-ahead reserve capacity is for compromising the real-time renewable power uncertainties. Constraints (11)-(12) ensure the generation of traditional DGs and gas turbines within the predefined limits considering reserve capacity. The voltage magnitude for all buses is regulated in (13) by setting minimum and maximum limits. In (14), the substation voltage can be determined by OLTC tap position and the step size of each tap position. Constraint (15) regulates the total operation number of OLTC tap since too many operations will accelerate the wear process of the transformer [21-23]. In (16), the reactive power of PV is described by the forecasted active PV power output and the power factor as defined in (17). The reactive power from capacitor banks is given in (18). The linearized DistFlow for distribution systems is presented in (19) and (20). This equation is obtained based on the assumption that i) losses are negligible, ii) the voltage at each bus is close to 1.0 p.u. and iii) the voltage at the reference bus is 1.0 p.u.. The power balance constraints for active and reactive power are in (21) and (22). The output of natural gas sources is constrained by (23). Equations (24) and (25) are the constraints on gas pressure, where, in distribution systems, the pressure of initial nodes is always higher than terminal nodes. In (26), Weymouth equation is used to characterize the relationship between gas flow and pressure. The gas flow is constrained in (27). The relationship between the gas turbine output and injected gas flow is given in (28). And equation (29) models nodal gas balancing.

$$
\begin{aligned}
& 0 \leq P_{s u b, t}^{S} \leq P_{s u b, \text { max }} \\
& 0 \leq Q_{\text {sub }, t}^{s} \leq Q_{\text {sub,max }} \\
& 0 \leq r_{\{\cdot\}, t}^{+} \leq R_{\{\cdot\}}^{+},\{\cdot\}=i_{e}, g t \\
& 0 \leq r_{\{\cdot, t}^{-} \leq R_{\{\cdot\}}^{-},\{\cdot\}=i_{e}, g t \\
& P_{\{\{, t}^{s}+r_{\{\{\}, t}^{+} \leq P_{\{\cdot\}, \max },\{\cdot\}=i_{e}, g t \\
& P_{\{\cdot\}, \min } \leq P_{\{\cdot, t, t}^{S}-r_{\{\cdot\}, t}^{-},\{\cdot\}=i_{e}, g t \\
& V_{b, \min } \leq V_{b, t}^{s} \leq V_{b, \max } \\
& V_{\text {sub }, t}^{s}=V_{\text {sub }}^{\text {ref }}+\delta^{\text {OLTC }} T P_{t}^{s, O L T C} \\
& \sum_{t \in T}\left|T P_{t}^{s, O L T C}-T P_{t-1,}^{s, O L T C}\right| \leq n T P_{\max }^{\text {OLTC }} \\
& -u_{P V} \omega_{j, t}^{P, s} \leq \omega_{j, t}^{Q, s} \leq u_{P V} \omega_{j, t}^{P, s} \\
& u_{P V}=\sqrt{\frac{1-P F_{P V, \min ^{2}}{ }^{2}}{P F_{P V, \text { min }}^{2}}} \\
& Q_{c b, t}^{s}=u_{c b, t}^{s} Q_{c b}^{c a p} \\
& V_{b, t}^{s, i n i}-V_{b, t}^{s, t e r}=\left(f_{l_{e}, t}^{a, s} r_{l_{e}}+f_{l_{e}, t}^{r, s} x_{l_{e}}\right) / V_{0} \\
& 0 \leq f_{l_{e}, t}^{\{\cdot\}, s} \leq f_{l_{e}, \max }^{\{\cdot,, s},\{\cdot\}=a, r \\
& \sum_{i_{e} \in I_{e}} P_{i_{e}, t}^{s}+\sum_{j \in J} \omega_{j, t}^{P, s}+\sum_{l_{e} \in L_{e}} f_{l_{e}, t}^{a, s, i n i}- \\
& \sum_{l_{e} \in L_{e}} f_{l_{e}, t}^{a, s, t e r}+\sum_{g t \in G T} P_{g t, t}^{s}=\sum_{k_{e} \in K_{e}} P_{k_{e}, t} \\
& \sum_{i_{e} \in I_{e}} Q_{i_{e}, t}^{s}+\sum_{j \in J} \omega_{j, t}^{Q, s}+\sum_{c b \in C B} Q_{c b, t}^{s}+\sum_{l_{e} \in L_{e}} f_{l_{e}, t}^{r, s, i n i} \\
& -\sum_{l_{e} \in L_{e}} f_{l_{e}, t}^{r, s, t e r}=\sum_{k_{e} \in K_{e}} Q_{k_{e}, t} \\
& P_{i_{g}, \min } \leq P_{i_{g}, t}^{S} \leq P_{i_{g}, \max } \\
& P r_{l_{g}, \text { min }}^{2} \leq P r_{l_{g}, t}^{s^{2}} \leq P r_{l_{g}, \text { max }}^{2} \\
& P r_{l_{g}, t}^{s, i n i} \geq P r_{l_{g}, t}^{s, t e} \\
& f_{l_{g}, t}^{s{ }^{2}}=\gamma_{l_{g}}\left(P r_{l_{g}, t}^{s, i n i^{2}}-P r_{l_{g}, t}^{s, t e r^{2}}\right) \\
& 0 \leq f_{l_{g}, t}^{s} \leq f_{l_{g}, \max } \\
& P_{g t, t}^{s}=c_{g t} f_{l g, g t}^{s} \\
& \sum_{i_{g} \in I_{g}} P_{i_{g}, t}^{s}+\sum_{l_{g} \in L_{g}} f_{l_{g}, t}^{s, i n i}-\sum_{l_{g} \in L_{g}} f_{l_{g}, t}^{s, t e r} \\
& =\sum_{k_{g} \in K_{g}} P_{k_{g}, t}+\sum_{g t \in G T} P_{g t, t}^{s}
\end{aligned}
$$

\section{B. Real-time $V V O$}

The real-time corrective dispatch is in the second stage considering renewable power uncertainty, which regulates voltage and generation output of traditional DGs and natural gas sources. Equations (30) and (31) limit the power transfer of substations. The regulated output of traditional DGs and gas turbines is given in (32). Electricity and gas load shedding constraint is in (33). Equation (34) is the constraint for voltages 
for all buses. The substation voltage is defined in (35). In (36), the regulated total operation number of OLTC tap is constrained. The reactive power of PV generators and capacitor banks are ensured in (37) and (38). DistFlow is applied again in the second stage describing power flow in (39) and (40). The active and reactive power balance constraints are in (41) and (42), respectively. The regulated output of natural gas sources is given in (43). For modelling gas flow, the Weymouth equation is presented from (44)-(47) with gas pressure constrained. Constraint (48) describes the relationship between the gas flow injection and gas turbine output. The aim of the regulation of traditional DGs and natural gas sources is to mitigate adverse effects from renewable output deviation, which is achieved by adjusting reserves for power capacity from (49) to (51). In (49), renewable output deviation should be within the up and down reserve limits. To address renewable power uncertainty, the participation factors $\eta_{i_{e}, t}$ and $\eta_{i_{g}, t}$ are defined in (50) and (51) to represent the regulation commitment by traditional DGs and natural gas sources. Finally, (52) presents the balancing condition of the gas system.

$$
\begin{aligned}
& 0 \leq P_{\text {sub }, t}^{r e} \leq P_{\text {sub, } \text { max }} \\
& 0 \leq Q_{\text {sub }, t}^{r e} \leq Q_{\text {sub,max }} \\
& P_{\{\cdot\}, t}^{r e}-r_{\{\cdot\}, t}^{-} \leq P_{\{\}, t}^{r e} \leq P_{\{\}, t}^{r e}+r_{\{\cdot\}, t}^{+},\{\cdot\}=i_{e}, g t \\
& 0 \leq P_{\{\cdot\}, t}^{l s} \leq P_{\{\cdot, \max }^{l s},\{\cdot\}=k_{e}, k_{g} \\
& V_{b, \min } \leq V_{b, t}^{r e} \leq V_{b, \max } \\
& V_{\text {sub }, t}^{r e}=V_{\text {sub }}^{r e f}+\delta^{\text {OLTC }} T P_{t}^{r e, O L T C} \\
& \sum_{t \in T}\left|T P_{t}^{r e, O L T C}-T P_{t-1,}^{r e, O L T C}\right| \leq n T P_{\max }^{\text {OLTC }} \\
& -u_{P V} \omega_{j, t}^{P, r e} \leq \omega_{j, t}^{Q, r e} \leq u_{P V} \omega_{j, t}^{P, r e} \\
& Q_{c b, t}^{r e}=u_{c b, t}^{r e} Q_{c b, t}^{r e f} \\
& V_{b}^{r e, i n i}-V_{b}^{r e, t e r}=\left(f_{l_{e}, t}^{a, r e} r_{l_{e}}+f_{l_{e}, t}^{r, r e} x_{l_{e}}\right) / V_{0} \\
& 0 \leq f_{l_{e}, t}^{\{\cdot, r e} \leq f_{l_{e}, \text { max }}^{\{\cdot\}},\{\cdot\}=a, r \\
& \sum_{i_{e} \in I_{e}} P_{i_{e}, t}^{r e}+\sum_{j \in J} \xi_{j, t}+\sum_{g t \in G T} P_{g t, t}^{s} \\
& =\sum_{k_{e} \in K_{e}} P_{k_{e}, t}+\Delta P_{k_{e}, t}-P_{k_{e}, t}^{l s} \\
& \sum_{i_{e} \in I_{e}} Q_{i_{e}, t}^{s}+\sum_{j \in J} \omega_{j, t}^{Q, s}+\sum_{c b \in C B} Q_{c b, t}^{s} \\
& +\sum_{l_{e} \in L_{e}} f_{l_{e}, t}^{r, s, i n i}-\sum_{l_{e} \in L_{e}} f_{l_{e}, t}^{r, s, t e r} \\
& =\sum_{k_{e} \in K_{e}} Q_{k_{e}, t} \\
& P_{i_{g}, \min } \leq P_{i_{g}, t}^{r e} \leq P_{i_{g}, \max } \\
& P r_{l_{g}, \text { min }}^{2} \leq P r_{l_{g}, t}^{r e^{2}} \leq P r_{l_{g}, \text { max }}^{2} \\
& P r_{l_{g}, t}^{r e, i n i} \geq P r_{l_{g}, t}^{r e, t e r} \\
& f_{l_{g}, t}^{r e}\left|f_{l_{g}, t}^{r e}\right|=\gamma_{l_{g}}\left(P r_{l_{g}, t}^{r e, i n i^{2}}-P r_{l_{g}, t}^{r e, t e r^{2}}\right) \\
& 0 \leq f_{l_{g}, t}^{r e} \leq f_{l_{g}, \max } \\
& P_{g t, t}^{r e}=c_{g t} f_{l_{g}, g t}^{r e}
\end{aligned}
$$

$$
\begin{gathered}
r_{i_{e}, t}^{-} \leq \eta_{i_{e}, t} \sum_{j \in J}\left(\omega_{j, t}^{s}-\xi_{j, t}\right) \leq r_{i_{e}, t}^{+},\{\cdot\}=i_{e}, g t \\
0 \leq \eta_{\{\cdot, t} \leq 1,\{\cdot\}=i_{e}, g t \\
\sum_{i_{e} \in I_{e}} \eta_{i_{e}, t}+\sum_{g t \in G T} \eta_{i_{g}, t}^{g t}=1 \\
\sum_{i_{g} \in I_{g}} P_{i_{g}, t}^{r e}+\sum_{l_{g} \in L_{g}} f_{l_{g}, t}^{r e, i n i}-\sum_{l_{g} \in L_{g}} f_{l_{g}, t}^{r e, t e r} \\
=\sum_{k_{g} \in K_{g}} P_{k_{g}, t}+\sum_{g t \in G T} P_{g t, t}^{r e}-P_{k_{g}, t}^{l s}
\end{gathered}
$$

\section{METHODOLOGY}

In this section, it proposes the methodology for solving the TS-VCO for IEGS. First, the simplification of the mathematical modelling is presented. The linearization is made through the transformation from the mixed-integer nonlinear programming (MINLP) to mixed-integer linear programming (MILP). Then the relaxation for the resulted MILP to a linear programming (LP) form is obtained. In part $\mathrm{B}$, the original problem is expressed in the abstract form. Part $\mathrm{C}$ models the ambiguity set for DRO for handling renewable power uncertainty. Part D presents dual formulations. Finally, parts E and F reformulate the dual formulation to semidefinite programming (SDP) formulation and employ CGA to solve it.

\section{A. Model Linearization}

To find the global optimum of TS-VCO, nonlinear equations in (2), (5), (15) and (36) should be linearised, where, auxiliary variables $\pi$ and $\vartheta$ are used to represent the absolute values. Equations (53)-(55) represent objective functions (2) and (5), and (56)-(59) represent constraints (15) and (36).

$$
\begin{gathered}
\pi=\left|V_{b, t}^{\cdot}-V_{b}^{r e f}\right|,\{\cdot\}=s, r e \\
\pi \geq V_{b, t}^{\cdot}-V_{b}^{r e f},\{\cdot\}=s, r e \\
\pi \geq V_{b}^{r e f}-V_{b, t}^{\cdot},\{\cdot\}=s, r e \\
\vartheta=\left|T P_{t}^{; O L T C}-T P_{t-1,}^{; O L T C}\right|,\{\cdot\}=s, r e \\
\vartheta \geq T P_{t}^{; O L T C}-T P_{t-1,}^{; O L T C},\{\cdot\}=s, r e \\
\vartheta \geq T P_{t-1,}^{, \text {OLTC }}-T P_{t}^{, \text {OLTC }},\{\cdot\}=s, r e \\
\sum_{t \in T} \vartheta \leq n T P_{\max }^{\text {OLTC }}
\end{gathered}
$$

The operation number of tap changers of OLTC and capacitor banks are modelled as discrete variables, i.e., $T P_{t}^{r e, O L T C}$ and $u_{c b, t}^{r e}$, which can be relaxed as continuous variables [22] for computational simplicity.

$$
\begin{gathered}
-16 \leq T P_{t}^{r e, O L T C} \leq 16 \\
0 \leq u_{c b, t}^{r e} \leq 1
\end{gathered}
$$

\section{B. Abstract Formulation}

The objective functions in the first and second stages in (1)(6) are formulated into the compact form in (62), where the first term represents (1) and the second term represents the expectation of (2). The first-stage problem determines the dayahead operation scheme before the realisation of renewable energy uncertainty. The second-stage problem 'min sup $E_{P}[Q(x, \xi)]$ ' in (62) is a bi-level optimization which determines 
the best operation when the worst-case scenario is selected from all uncertainty realizations.

$$
\begin{gathered}
\min _{x \in X} c^{\prime} x+\sup _{P f \in D \xi \xi=\xi_{j}} E_{P}[Q(x, \xi)] \\
\text { s.t. } A x \leq b, \\
Q(x, \xi)=\min _{y} f^{\prime} y \\
\text { s.t. } E x+F y+G \xi \leq h,
\end{gathered}
$$

Where, the constraints of the first and second stages are in (64) and (65), and vector $f$ in (64) is the coefficient of (4).

\section{DRO and Ambiguity Set}

Ambiguity set constructed by historical statistical data is used to model uncertainty distribution. In this paper, the momentbased ambiguity set is used to incorporate mean and covariance information to represent all possible distributions, which is shown in (66). The ambiguity set ensures i) the integral of the probability distribution of $\xi$ is 1 and ii) all distributions have common mean and covariance.

$$
D=\left\{\begin{array}{l|c}
f(\xi) & \begin{array}{c}
\mathrm{P}\{\xi\}=1 \\
\mathrm{E}\{\xi\}=\mu \\
\mathrm{E}\left\{\xi(\xi)^{\prime}\right\}=\Sigma+\mu(\mu)^{\prime}
\end{array}
\end{array}\right\}
$$

\section{Second-stage Dual Formulation}

In (66), the probability densities are the variables to be determined in the proposed ambiguity set. Due to the characteristics of DRO which considers all possible ambiguous distributions that immunize the worst-case distribution, equations (62)-(65) contain an infinite number of variables and a finite number of constraints. A dual formulation is required to transform the original problem into a dualized form with a finite number of variables and an infinite number of constraints.

Accordingly, the original second-stage problem in (62), i.e., $\sup E_{P}[Q(x, \xi)]$ can be transformed into (67), which is an $P f \in D$

explicit primal form. Where, $\psi_{0}, \psi_{j}$ and $\Psi_{j k}$ are dual variables and the probability density function is represented by $P f(\xi)$.

$$
\begin{gathered}
S(x)^{\text {primal }}=\max _{P f \in D_{\xi}} \int_{\Xi} Q(x, \xi) P f(\xi) d \xi \\
\text { s.t. } P f(\xi) \geq 0, \forall \xi \in \Xi \\
\int_{\Xi} P f(\xi) d \xi=1 \\
\int_{\Xi} \xi^{m} P f(\xi) d \xi=\mu_{m}, m=1,2, \ldots, \Xi \\
\int_{\Xi} \xi^{m} \xi^{n} P f(\xi) d \xi=\Sigma_{m n}+\mu_{m} \mu_{n}, m, n=1,2, \ldots, \Xi
\end{gathered}
$$

Meanwhile, problem (72)-(73) has a finite number of variables, which is easier to solve than the original primal form in (67). Where, $\left\langle\Psi^{\prime} \Theta\right\rangle$ represents the trace of matrix $\Psi^{\prime} \Theta$ and $\Theta=\Sigma+\mu(\mu)^{\prime}$. Therefore, the new compact form of TS-VCO is given in (74).

$$
\begin{gathered}
S(x)^{\text {dual }}=\min _{\Psi, \psi, \psi_{0}}\left\langle\Psi^{\prime} \Theta\right\rangle+\psi^{\prime} \mu+\psi_{0} \\
\text { s.t. }(\xi)^{\prime} \Psi \xi+\psi^{\prime} \xi+\psi_{0} \geq Q(x, \xi) \\
\forall \xi \in \Xi \\
\min _{x \in X} c^{\prime} x+S(x)^{d u a l}
\end{gathered}
$$

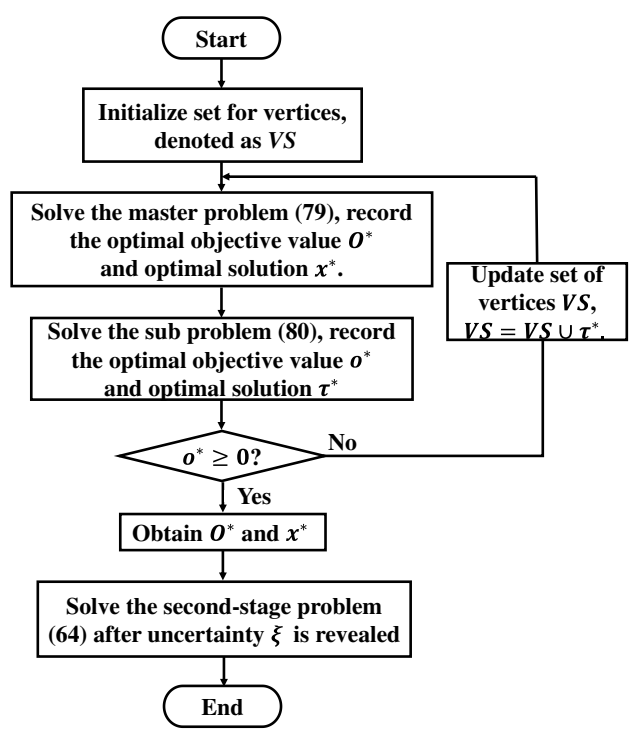

Fig. 1. Flowchart of constraint generation algorithm

\section{E. SDP Reformulation}

Hence, the closed-form of the original problem is required to ensure tractability. Equation (75) is obtained with a newly introduced dual variable $\tau$. Equation (72) can be rewritten as a positive quadratic function (77) based on the new dual variable

$$
\begin{gathered}
\max _{u \in V S} \tau^{\prime}(b-E x-G \xi) \\
V S=\left\{\tau \mid F^{\prime} \tau=f, \tau \leq 0\right\} \\
(\xi)^{\prime} \Psi \xi+\left(\psi+G^{\prime} \tau^{i}\right)^{\prime} \xi+\psi_{0}-(h-E x) \tau^{i} \geq 0 \\
\forall \xi \in \Xi, i=1,2, \ldots, N_{v}
\end{gathered}
$$

Written in the compact matrix form, the original TS-VCO is finally expressed in the SDP form:

$$
\begin{gathered}
\min _{x, \Psi, \psi, \psi_{0}} c^{\prime} x+\left\langle\Psi^{\prime} \Theta\right\rangle+\psi^{\prime} \mu+\psi_{0} \\
{\left[\begin{array}{c}
\xi \\
1
\end{array}\right]^{\prime}\left[\begin{array}{cc}
\Psi & \frac{1}{2}\left(\psi+G^{\prime} \tau^{i}\right) \\
\frac{1}{2}\left(\psi+G^{\prime} \tau^{i}\right)^{\prime} & \psi_{0}-(h-E x)^{\prime} \tau^{i}
\end{array}\right]\left[\begin{array}{l}
\xi \\
1
\end{array}\right] \geq 0} \\
\forall \xi \in \Xi, i=1,2, \ldots, N_{v}, x \in X, \forall \tau^{i} \in V S
\end{gathered}
$$

\section{F. Constraint Generation Algorithm}

Although the SDP problem in (78) is tractable, the large computation burden caused by the vast number of constraints of (78) with infinite $V S$ cardinality is still a big challenge. CGA can be used to relax all constraints by enumerating a subset of vertices and finally searching for the vertex set which constitutes SDP constraints [24, 25]. CGA enumerates more constraints in each iteration and checks whether they are fulfilled by (77). The optimality gap is used to ensure CGA terminates in a finite number of iterations when the difference between updated upper and lower bound is within the tolerance. Equations (79) and (80) are the master and subproblems.

The initial set for all the vertices is set in the first step. Then the master and sub problems are solved in turn. At each iteration, the optimal objective value is checked if it is above 0 . If it is not, the set of vertices is updated to incorporate more vertices. When the terminal condition is satisfied, record the optimal value and optimal solution. Then the second-stage problem can be solved based on an expected manner. The detailed steps of the proposed CGA are given in Fig. 1. 


$$
\begin{gathered}
\min _{x, \Psi, \psi, \psi_{0}} c^{\prime} x+\left\langle\Psi^{\prime} \Theta\right\rangle+\psi^{\prime} \mu+\psi_{0} \\
\Psi\left[\begin{array}{c}
\xi \\
1
\end{array}\right]^{\prime}\left[\begin{array}{cc}
\Psi & \frac{1}{2}\left(\psi+G^{\prime} \tau^{i}\right) \\
\frac{1}{2}\left(\psi+G^{\prime} \tau^{i}\right)^{\prime} & \psi_{0}-(h-E x)^{\prime} \tau^{i}
\end{array}\right]\left[\begin{array}{l}
\xi \\
1
\end{array}\right] \geq 0 \\
\forall \xi \in \Xi, i=1,2, \ldots, N_{v}, x \in X, \forall \tau^{i} \in V S \\
\left(\xi_{s}\right)^{\prime} \Psi \xi_{s}+\psi^{\prime} \xi_{s}+\psi_{0}-\left(h-E x-G \xi_{s}\right)^{\prime \tau} \geq 0 \\
\text { s.t. } \forall \xi \in \Xi, \tau \in V S
\end{gathered}
$$

\section{CASE STUDIES}

As shown in Fig. 2, the proposed TS-VCO and CGA are firstly verified on a modified IEEE 33-bus system connected with a 6-node gas system in radial topology. A gas turbine connects the two separated systems, which generates electricity using natural gas. To testify the effectiveness of the TS-VCO in different conditions, comparison between 8 scenarios is considered and the details are given in TABLE II. Cases 1-3 are used to compare mathematical performance of different optimization methods. Cases 3-5 compare the impact of varying optimization priorities on voltage deviation and economic performance. The impact of PV penetration and capacitor bank capacity are analysed in cases 3,6 and 7. Case 8 studies the scenario without natural gas connection.

In the electricity system shown in Fig. 2, it contains i) 3 traditional DGs connected with bus 13, 21 and 28 , ii) 3 PV systems connected with bus 11,16 and 22 with each capacity of $200 \mathrm{kVA}$, and iii) 7 capacitor banks which have the same capacity of 30kVar. The substation transformer has 32 tap positions with a step size of $0.00,3$ which ranges from -16 to 16. The maximum allowed operation number between two continuous-time slots for OLTC is set as 3 . The voltage limit on each bus is set between 0.95 p.u. and 1.05 p.u.. The gas system has 6 nodes, containing 2 natural gas sources and 3 gas loads. The conversion factor 3.313 is used to convert $\$ / \mathrm{kcf}$ to $\$ / \mathrm{MWh}$ when simultaneously considering electricity and gas load shedding in IEGS. This paper uses $100 \$ / \mathrm{MWh}$ and $120 \$ / \mathrm{kcf}$ $(400 \$ / \mathrm{MWh})$ as the shedding cost for electricity and gas load, respectively. The detailed parameters of natural gas sources and traditional DGs are given in TABLE III and IV.

\section{A. Studies on Economic Performance}

The economic performance for all cases is analysed first, presented in TABLE V and TABLE VI. The cost of the first and second stages are presented in TABLE V. Case 5 has the highest total cost (\$30111) while case 4 has the lowest cost (\$25398) since the optimization priority of case 5 focuses on minimizing the voltage deviation while gives less focus on economic performance. Case 4 considers $75 \%$ of objective weighting on economic performance, which leads to $\$ 4713$ less total cost than case 5. Cases 1-3 have the same optimization priority on subobjectives and the same IEGS configuration. Case 1 provides a total cost of $\$ 27343$, which is $5.8 \%$ and $2.8 \%$ less than case 2 and 3. The advantage of DRO is the less conservatism when modelling PV output uncertainty compared with RO, which is reflected in the $2.9 \%$ less cost of case 3 compared with case 2 . Compared with case 3 , the PV capacity is doubled and quadrupled in cases 6 and 7 , which address the high generation of traditional DGs and natural gas sources. However, compared with case 3, case 6 and 7 cause higher second-stage cost due to the penalty cost of PV output deviation in the first and second

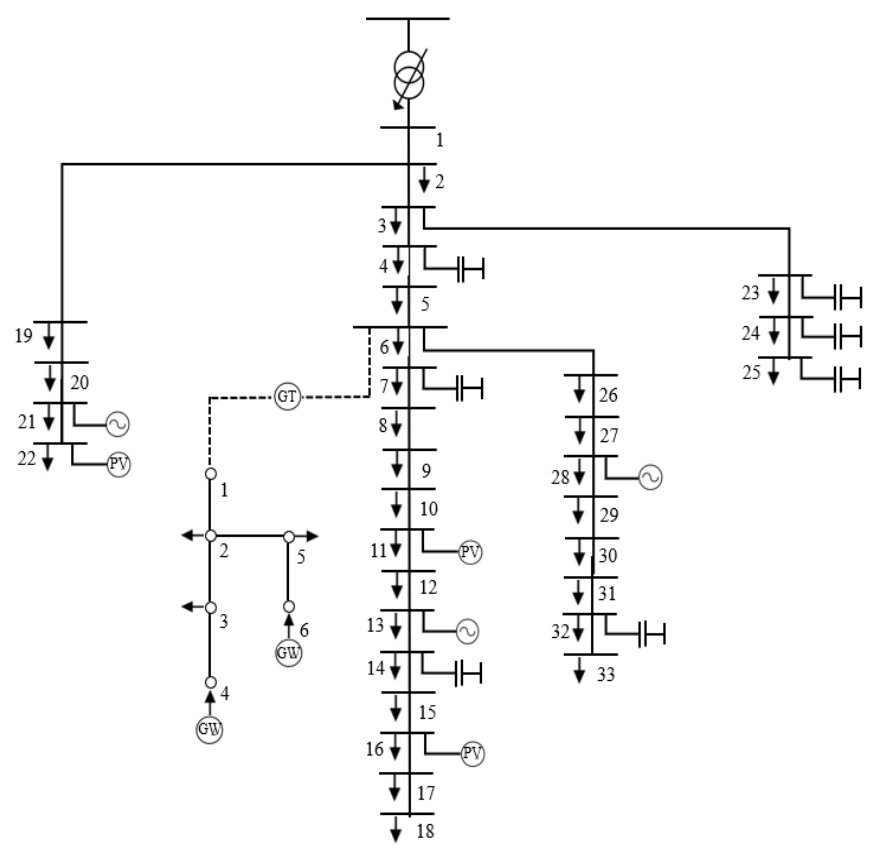

Fig. 2. A modified IEEE 33-bus system and a 6-node gas system.

TABLE I

CASE ILLUSTRATION

\begin{tabular}{ccccc}
\hline \hline $\begin{array}{c}\text { Case } \\
\text { No. }\end{array}$ & $\begin{array}{c}\text { Weighting } \\
\text { coefficients }\end{array}$ & $\begin{array}{c}\text { Optimization } \\
\text { method }\end{array}$ & $\begin{array}{c}\text { PV system } \\
\text { capacity } \\
(\mathrm{kVA})\end{array}$ & $\begin{array}{c}\text { Connected } \\
\text { to gas } \\
\text { system }\end{array}$ \\
\hline 1 & $w^{V}=0.5$ & Deterministic & 200 & Yes \\
2 & $w^{V}=0.5$ & Robust & 200 & Yes \\
3 & $w^{V}=0.5$ & DRO & 200 & Yes \\
4 & $w^{V}=0.25$ & DRO & 200 & Yes \\
5 & $w^{V}=0.75$ & DRO & 200 & Yes \\
6 & $w^{V}=0.5$ & DRO & 400 & Yes \\
7 & $w^{V}=0.5$ & DRO & 800 & Yes \\
8 & $w^{V}=0.5$ & DRO & 200 & No \\
\hline \hline
\end{tabular}

TABLE II

PARAMETERS OF NATURAL GAS SOURCES

\begin{tabular}{cccc}
\hline \hline Node No. & $\begin{array}{c}P_{i_{g}, \min } \\
(\mathrm{kcf} / \mathrm{h})\end{array}$ & $\begin{array}{c}P_{i_{g}, \max } \\
(\mathrm{kcf} / \mathrm{h})\end{array}$ & $\begin{array}{c}\lambda_{i_{g}} \\
(\$ / \mathrm{kcf})\end{array}$ \\
\hline 4 & 1000 & 6000 & 2.2 \\
6 & 1000 & 3000 & 2 \\
\hline \hline
\end{tabular}

TABLE III

GENERATOR PARAMETERS

\begin{tabular}{ccccccc}
\hline \hline $\begin{array}{c}\text { Bus } \\
\text { No. }\end{array}$ & $\begin{array}{c}P_{i_{e}, \max } \\
(\mathrm{MW})\end{array}$ & $\begin{array}{c}P_{i_{e}, \min } \\
(\mathrm{MW})\end{array}$ & $\begin{array}{c}R_{i}^{+}, R_{i}^{-} \\
(\mathrm{MW})\end{array}$ & $\begin{array}{c}a_{i} \\
\left(\$ / \mathrm{MW}^{2}\right)\end{array}$ & $\begin{array}{c}b_{i} \\
(\$ / \mathrm{MW})\end{array}$ & $\begin{array}{c}c_{i} \\
(\$)\end{array}$ \\
\hline 13 & 1.2 & 0.3 & 0.2 & 6000 & 7100 & 6200 \\
21 & 1.2 & 0.3 & 0.2 & 4500 & 10500 & 4000 \\
28 & 1.0 & 0.1 & 0.2 & 4500 & 10500 & 4000 \\
\hline \hline
\end{tabular}

stage. Without support from the gas system, case 8 yields $4.7 \%$ more cost than case 3 . It is because the generation cost from natural gas sources is lower than that of traditional DGs and the pipeline capacity is set enough for large gas flow.

In TABLE VI, energy supply cost at the substation from the upper energy market in the first stage, as well as the load shedding cost in the second stage, are investigated. The influence on the economic result caused by the weighting coefficient, optimization method, PV capacity and IEGS structure are investigated. As for energy supply cost, only case 4 has the result of $\$ 12880$ and the other cases are all $\$ 14000$. As for the load shedding cost, case 4 has the minimum cost 
TABLE IV

COST OF EACH STAGE FOR CASE $1-8$

\begin{tabular}{|c|c|c|c|c|}
\hline Economic result & Case 1 & Case 2 & Case 3 & Case 4 \\
\hline First-stage cost $(\$)$ & 27343 & 28929 & 27002 & 24523 \\
\hline $\begin{array}{l}\text { Expected Second- } \\
\text { stage cost }(\$)\end{array}$ & 0 & 0 & 1105 & 875 \\
\hline Total cost $(\$)$ & 27343 & 28929 & 28107 & 25398 \\
\hline Economic result & Case 5 & Case 6 & Case 7 & Case 8 \\
\hline First-stage cost $(\$)$ & 28425 & 25305 & 24196 & 27891 \\
\hline $\begin{array}{l}\text { Expected Second- } \\
\text { stage cost }(\$)\end{array}$ & 1686 & 1200 & 1443 & 1560 \\
\hline Total cost $(\$)$ & 30111 & 26505 & 25639 & 29451 \\
\hline
\end{tabular}

TABLE V

ENERGY SUPPLY AND LOAD SHEDDING COST FOR CASE 1-8

\begin{tabular}{|c|c|c|c|c|}
\hline Economic result & Case 1 & Case 2 & Case 3 & Case 4 \\
\hline $\begin{array}{l}\text { Energy supply } \\
\text { cost }(\$)\end{array}$ & 14000 & 14000 & 14000 & 12880 \\
\hline $\begin{array}{l}\text { Load shedding } \\
\text { cost }(\$)\end{array}$ & 0 & 0 & 1084 & 210 \\
\hline Economic result & Case 5 & Case 6 & Case 7 & Case 8 \\
\hline $\begin{array}{l}\text { Energy supply } \\
\text { cost }(\$)\end{array}$ & 14000 & 14000 & 14000 & 14000 \\
\hline $\begin{array}{l}\text { Load shedding } \\
\text { cost }(\$)\end{array}$ & 1390 & 604 & 492 & 1001 \\
\hline
\end{tabular}

(\$210) and case 5 has the highest cost $(\$ 1390)$. Case 4 and 5 yield the lowest and highest result, which indicates the weighting coefficient set on the sub-objectives has the greatest influence on the optimization result compared with optimization method, PV capacity and IEGS structure. It can be seen that apart from cases 1 and 2 of a single-stage framework, both the energy supply cost and load shedding cost in case 4 are the lowest since TS-VCO takes $75 \%$ weight on economic performance-oriented sub-objective. In cases 6 and 7, load shedding cost is also relatively low since PV capacity is adequately sufficient.

\section{B. Studies on Voltage Profile}

The voltage profiles of 33 buses in 24 time periods for cases 3, 6 and 7 with different capacity of PV systems are shown in Fig. 3-5. The red dotted curve is the mean voltage profile among all buses for clearer presentation and comparison. In case 3, with the least PV capacity connected, the voltage level ranges from 0.963 p.u. to 1.016 p.u.. With the higher PV connection in case 6, i.e., 200kVA more capacity of each PV, the voltage profile has been improved by $1 \%$. In case 7 , the voltage level reaches up to 1.032 p.u. when the PV capacity is $800 \mathrm{kVA}$, which causes voltage improvement by $1.5 \%$ compared with case 3. The comparison between case 3, 6 and 7 shows the increase of voltage level for all buses with the increasing PV penetration. The PV systems not only provide active power support but reactive power support, which will lead to the reduction of power losses in the real world. In Fig. 6, without connecting the gas system, the voltage profile of case 8 ranges between 0.96 p.u. and 1.00 p.u., which is lower than in case 3 .

Cases 3, 6, 7 and 8 are used to study the impact of PV uncertainty on voltage deviations with different methods. In Fig. 7 , voltage profile from the deterministic optimization is the

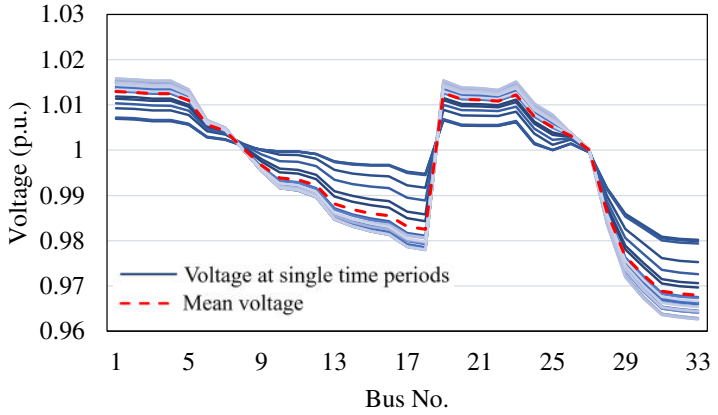

Fig. 3. Expected real-time voltage profiles for case 3 .

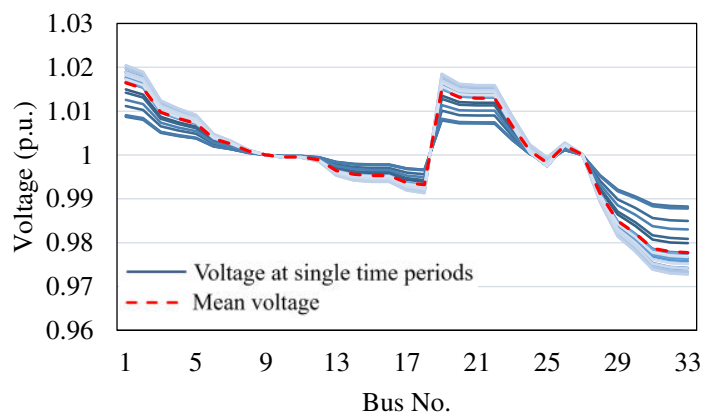

Fig. 4. Expected real-time profiles for case 6

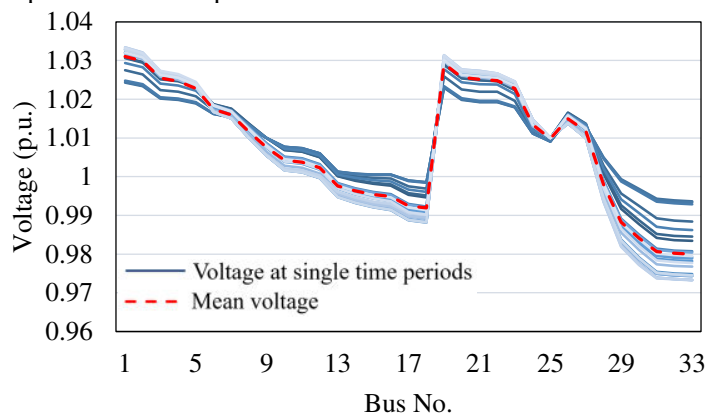

Fig. 5. Expected real-time profiles for case 7.

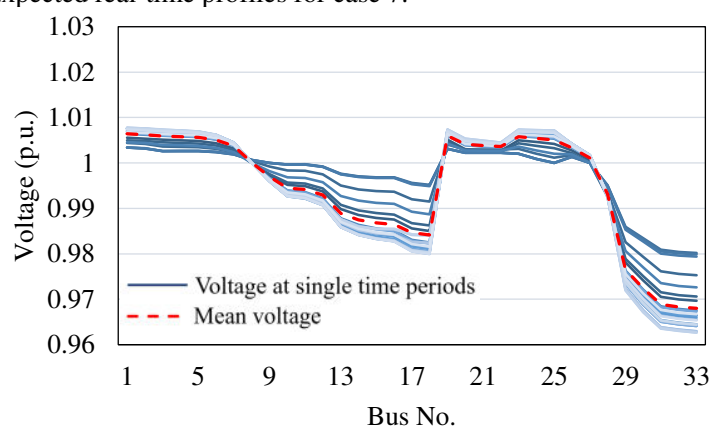

Fig. 6. Expected real-time voltage profiles for case 8 .

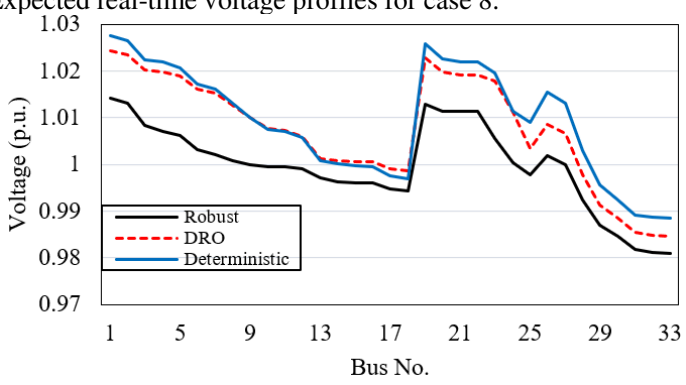

Fig. 7. Voltage profiles for case 1,2 and 3 at 20 th time period.

highest, whilst that from RO is the lowest. The voltage difference is approximately 0.01 p.u., i.e., $0.13 \mathrm{kV}$. Compared to 
the deterministic approach, DRO generates more conservative results. Compared with RO, DRO mitigates the conservatism by 0.008 p.u., which considers the worst uncertainty distribution instead of the worst-case PV uncertainty of RO.

\section{Studies on OLTC}

The OLTC tap position for 24 time periods is given in Fig. 8. With the highest PV capacity connected, the OLTC tap position remains the highest in case 7 , which starts from +10 position and maintains at +11 position from the 8 th hour to 24 th hour. With lower PV capacity, OLTC tap position in cases 3 and 6 are at a relatively lower level since the system voltage level is low and can be maintained in an acceptable range without a large deviation from nominal voltage. In case 8 , the tap position ranges from +1 to +3 and the maximum tap position deviation is 1 , i.e., from +1 to +2 or +2 to +3 . The reason is without the gas system connected, there is no power support converted from the gas flow, which will not raise the voltage level.

\section{CONCLUSION}

This paper proposes a multi-objective optimization for minimizing both operation cost and voltage deviation of IES considering renewable power uncertainty. A two-stage datadriven DRO approach is used to solve the TS-VCO with dual and SDP formulations to ensure computational tractability. The reformulated TS-VCO is solved by CGA with master and subproblems. The key findings from the case studies are :

- Based on a large amount of moment information, DRO produces less conservative results compared with $\mathrm{RO}$, more effective for maintaining voltage deviation and reducing operation cost considering renewable power uncertainty.

- The interdependency between electricity and gas systems reduces significant operation cost and voltage rise.

- The proposed TS-VCO is effective in maintaining voltage and saving operation cost considering PV uncertainty.

This work can benefit integrated system operators with powerful operation tool to manage the systems with fewer costs but integrate more renewable energy. To end customers, they can enjoy enhanced energy supply quality at low costs.

\section{REFERENCES}

[1]B. A. d. Souza and A. M. F. d. Almeida, "Multiobjective Optimization and Fuzzy Logic Applied to Planning of the Volt/Var Problem in Distributions Systems," IEEE Transactions on Power Systems, vol. 25, no. 3, pp. 1274-1281, 2010, doi: 10.1109/TPWRS.2010.2042734.

[2]H. Ahmadi, J. R. Martí, and H. W. Dommel, "A Framework for Volt-VAR Optimization in Distribution Systems," IEEE Transactions on Smart Grid, vol. 6, no. 3, pp. 1473-1483, 2015, doi: 10.1109/TSG.2014.2374613.

[3] A. Padilha-Feltrin, D. A. Q. Rodezno, and J. R. S. Mantovani, "Volt-VAR Multiobjective Optimization to Peak-Load Relief and Energy Efficiency in Distribution Networks," IEEE Transactions on Power Delivery, vol. 30, no. 2, pp. 618-626, 2015, doi: 10.1109/TPWRD.2014.2336598.

[4]R. Zafar, J. Ravishankar, J. E. Fletcher, and H. R. Pota, "Optimal Dispatch of Battery Energy Storage System using Convex Relaxations in Unbalanced Distribution Grids," IEEE Transactions on Industrial Informatics, pp. 1-1, 2019, doi: 10.1109/TII.2019.2912925.

[5] K. Kumar, S. Satsangi, and G. B. Kumbhar, "Extension of life of distribution transformer using Volt-VAr optimisation in a distribution system," IET Generation, Transmission \& Distribution, vol. 13, no. 10, pp. 1777-1785, 2019, doi: 10.1049/iet-gtd.2018.5746.

[6]F. U. Nazir, B. C. Pal, and R. A. Jabr, "A Two-Stage Chance Constrained Volt/Var Control Scheme for Active Distribution Networks With Nodal Power Uncertainties," IEEE Transactions on Power Systems, vol. 34, no. 1, pp. 314325, 2019, doi: 10.1109/TPWRS.2018.2859759.
[7]Y. P. Agalgaonkar, B. C. Pal, and R. A. Jabr, "Stochastic Distribution System Operation Considering Voltage Regulation Risks in the Presence of PV Generation," IEEE Transactions on Sustainable Energy, vol. 6, no. 4, pp. 13151324, 2015, doi: 10.1109/TSTE.2015.2433794.

[8]T. Ding, S. Liu, W. Yuan, Z. Bie, and B. Zeng, "A Two-Stage Robust Reactive Power Optimization Considering Uncertain Wind Power Integration in Active Distribution Networks," IEEE Transactions on Sustainable Energy, vol. 7, no. 1, pp. 301-311, 2016, doi: 10.1109/TSTE.2015.2494587.

[9]A. Martinez-Mares and C. R. Fuerte-Esquivel, "A Unified Gas and Power Flow Analysis in Natural Gas and Electricity Coupled Networks," IEEE Transactions on Power Systems, vol. 27, no. 4, pp. 2156-2166, 2012, doi: 10.1109/TPWRS.2012.2191984.

[10] C. Unsihuay-Vila, J. W. Marangon-Lima, A. C. Z. d. Souza, I. J. PerezArriaga, and P. P. Balestrassi, "A Model to Long-Term, Multiarea, Multistage, and Integrated Expansion Planning of Electricity and Natural Gas Systems," IEEE Transactions on Power Systems, vol. 25, no. 2, pp. 1154-1168, 2010, doi: 10.1109/TPWRS.2009.2036797.

[11] C. He, L. Wu, T. Liu, and M. Shahidehpour, "Robust Co-Optimization Scheduling of Electricity and Natural Gas Systems via ADMM," IEEE Transactions on Sustainable Energy, vol. 8, no. 2, pp. 658-670, 2017, doi: 10.1109/TSTE.2016.2615104.

[12] M. Qadrdan, J. Wu, N. Jenkins, and J. Ekanayake, "Operating Strategies for a GB Integrated Gas and Electricity Network Considering the Uncertainty in Wind Power Forecasts," IEEE Transactions on Sustainable Energy, vol. 5, no. 1, pp. 128-138, 2014, doi: 10.1109/TSTE.2013.2274818.

[13] Y. He, M. Shahidehpour, Z. Li, C. Guo, and B. Zhu, "Robust Constrained Operation of Integrated Electricity-Natural Gas System Considering Distributed Natural Gas Storage," IEEE Transactions on Sustainable Energy, vol. 9, no. 3, pp. 1061-1071, 2018, doi: 10.1109/TSTE.2017.2764004.

[14] P. Zhao, C. Gu, D. Huo, Y. Shen, and I. Hernando-Gil, "Two-Stage Distributionally Robust Optimization for Energy Hub Systems," IEEE Transactions on Industrial Informatics, vol. 16, no. 5, pp. 3460-3469, 2020, doi: 10.1109/TII.2019.2938444.

[15] P. Zhao, C. Gu, and D. Huo, "Two-Stage Coordinated Risk Mitigation Strategy for Integrated Electricity and Gas Systems under Malicious False Data Injections," IEEE Transactions on Power Systems, pp. 1-1, 2020, doi: 10.1109/TPWRS.2020.2986455

[16] P. Zhao, H. Wu, C. Gu, and I. Hernando-Gil, "Optimal home energy management under hybrid photovoltaic-storage uncertainty: a distributionally robust chance-constrained approach," IET Renewable Power Generation, vol. 13, no. 11, pp. 1911-1919, 2019, doi: 10.1049/iet-rpg.2018.6169.

[17] X. Lu, K. W. Chan, S. Xia, X. Zhang, G. Wang, and F. Li, "A Model to Mitigate Forecast Uncertainties in Distribution Systems Using the Temporal Flexibility of EVAs," IEEE Transactions on Power Systems, vol. 35, no. 3, pp. 2212-2221, 2020, doi: 10.1109/TPWRS.2019.2951108.

[18] X. Lu, K. W. Chan, S. Xia, B. Zhou, and X. Luo, "Security-Constrained Multiperiod Economic Dispatch With Renewable Energy Utilizing Distributionally Robust Optimization," IEEE Transactions on Sustainable Energy, vol. 10, no. 2, pp. 768-779, 2019, doi: 10.1109/TSTE.2018.2847419. [19] L. Wu, C. He, X. Zhang, and T. Liu, "Distributionally Robust Scheduling of Integrated Gas-Electricity Systems with Demand Response," IEEE Transactions on Power Systems, pp. 1-1, 2019, doi: 10.1109/TPWRS.2019.2907170.

[20] F. Shahnia, S. Rajakaruna, and A. Ghosh, Static compensators (STATCOMs) in power systems. Springer, 2015.

[21] F. Mancilla-David, A. Angulo, and A. Street, "Power Management in Active Distribution Systems Penetrated by Photovoltaic Inverters: A DataDriven Robust Approach," IEEE Transactions on Smart Grid, pp. 1-1, 2019, doi: 10.1109/TSG.2019.2951086.

[22] M. B. Liu, C. A. Canizares, and W. Huang, "Reactive Power and Voltage Control in Distribution Systems With Limited Switching Operations," IEEE Transactions on Power Systems, vol. 24, no. 2, pp. 889-899, 2009, doi: 10.1109/TPWRS.2009.2016362.

[23] D. Choeum and D. Choi, "OLTC-Induced False Data Injection Attack on Volt/VAR Optimization in Distribution Systems," IEEE Access, vol. 7, pp. 34508-34520, 2019, doi: 10.1109/ACCESS.2019.2904959.

[24] E. Delage and Y. Ye, "Distributionally robust optimization under moment uncertainty with application to data-driven problems," Operations research, vol. 58, no. 3, pp. 595-612, 2010.

[25] D. Bertsimas, X. V. Doan, K. Natarajan, and C.-P. Teo, "Models for minimax stochastic linear optimization problems with risk aversion," Mathematics of Operations Research, vol. 35, no. 3, pp. 580-602, 2010. 


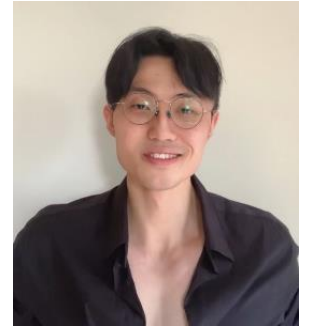

Pengfei Zhao (S'18) was born in Beijing, China. He received the double B.Eng. degree from the University of Bath, U.K., and North China Electric Power University, China, in 2017. He is currently pursuing the Ph.D. degree with the Department of Electronic and Electrical Engineering, University of Bath, U.K. He was a visiting Ph.D. student at Smart Grid Operations and Optimization Laboratory (SGOOL), Tsinghua University, Beijing, China in 2019. His research interests include the operation and planning of integrated energy systems considering inherent uncertainties.

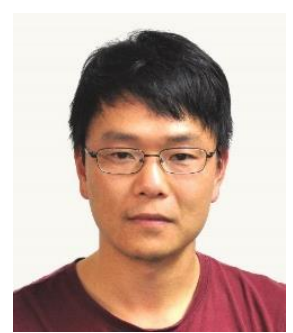

Chenghong Gu (M'14) was born in Anhui province, China. He received the Master's degree from the Shanghai Jiao Tong University, Shanghai, China, in 2007 in electrical engineering. He received the Ph.D. degree from the University of Bath, U.K. He is currently a Lecturer and EPSRC Fellow with the Department of Electronic and Electrical Engineering, University of Bath. His major research interest is in multi-vector energy system, smart grid, and power economics.

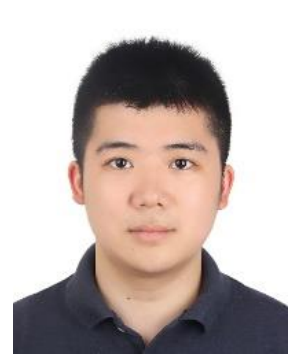

Yue Xiang (S'12-M'16) received the B.S. and Ph.D degrees from Sichuan University, China, in 2010 and 2016, respectively. From 2013 to 2014, he was a joint Ph.D. student at the Department of Electrical Engineering and Computer Science, University of Tennessee, Knoxville, US, a visiting scholar at the Department of Electronic and Electrical Engineering, University of Bath, UK in 2015, and also a visiting researcher at Department of Electrical and Electronic Engineering, Imperial College London, UK in 2019-2020. Now he is an associate professor in the College of Electrical Engineering, Sichuan University, China. His main research interests are distribution network planning and optimal operation, power economics, electric vehicle integration and smart grids.

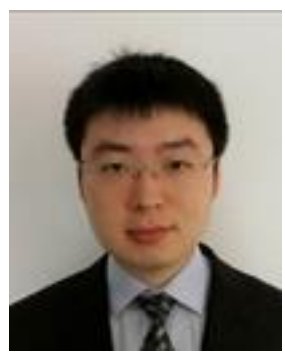

Xin Zhang received his B.Eng. degree in automation from Shandong University, China, in 2006; his M.S. and Ph.D. degrees in electrical power engineering from The University of Manchester, U.K., in 2007 and 2010 respectively. He is a senior lecturer in energy systems at Cranfield University, U.K. He previously worked for electricity national control at the National Grid, U.K. His main research interests include power system operation, renewable energy integration, and low carbon technologies in energy systems.

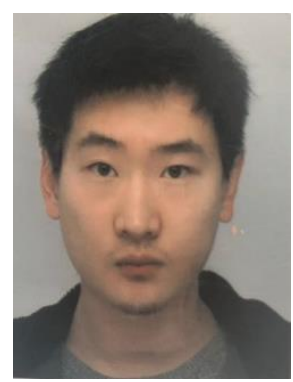

Yichen Shen was born in Hebei, China. He received the double bachelor degree in electrical engineering from North China Electric Power University, China and University of Bath, U.K. in 2017. He is currently working towards the Ph.D. the University of Bath. His major research scope is resilient multi energy systems.

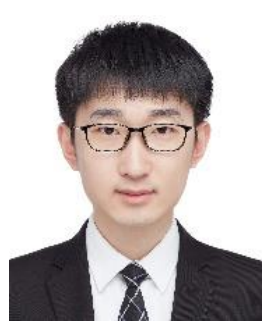

Shuangqi Li was born in Beijing, China. He received the B.Eng. degree in vehicle engineering from Beijing Institute of Technology, Beijing, China, in 2018. He worked as a research assistant at the National Engineering Laboratory for Electric Vehicles, Beijing Institute of Technology from 2018 to 2019. Currently, he is pursuing the Ph.D. degree at the Department of Electronic and Electrical Engineering, University of Bath. His major research interest is the big data analysis, deep-learning algorithm, operation and planning of smart grid system and V2G service. 